Article

\title{
Tuned Bis-Layered Supported Ionic Liquid Catalyst (SILCA) for Competitive Activity in the Heck Reaction of Iodobenzene and Butyl Acrylate
}

\author{
Nemanja Vucetic ${ }^{1, *(1)}$, Pasi Virtanen ${ }^{1}$, Ayat Nuri ${ }^{1,2}$, Andrey Shchukarev ${ }^{3}{ }^{1}$, \\ Jyri-Pekka Mikkola ${ }^{1,3}$ and Tapio Salmi ${ }^{1}$ \\ 1 Laboratory of Industrial Chemistry and Reaction Engineering, Johan Gadolin Process Chemistry Centre, \\ Åbo Akademi University, Biskopsgatan 8, FI-20500 Turku/Åbo, Finland; pasi.virtanen@abo.fi (P.V.); \\ ayatnuri@gmail.com (A.N.); jyri-pekka.mikkola@abo.fi (J.-P.M.); tapio.salmi@abo.fi (T.S.) \\ 2 Department of Applied Chemistry, Faculty of Science, University of Mohaghegh Ardabili, \\ Ardabil 56199-11367, Iran \\ 3 Technical Chemistry, Department of Chemistry, Umeå University, SE-90187 Umeå, Sweden; \\ andrey.shchukarev@umu.se \\ * Correspondence: nvucetic@abo.fi; Tel.: +358-41-703-7071
}

Received: 17 June 2020; Accepted: 19 August 2020; Published: 22 August 2020

\begin{abstract}
A thorough experimental optimization of supported ionic liquid catalyst (SILCA) was performed in order to obtain a stable and efficient catalyst for the Heck reaction. Out of fifteen proposed structures, propyl imidazolium bromide-tetramethylguanidinium pentanoate modified $\mathrm{SiO}_{2}$ loaded with $\mathrm{PdCl}_{2}$ appeared to be the most stable and to have a good activity in the reaction between butylacrylate and iodobezene, resulting in a complete conversion in $40 \mathrm{~min}$ at $100{ }^{\circ} \mathrm{C}$, in four consecutive experiments. This study elucidated on the stability of the catalytic system with an ionic liquid layer during the catalyst synthesis but also under reaction conditions. In the bis-layered catalyst, the imidazolium moiety as a part of internal layer, brought rigidity to the structure, while in external layer pentanoic acid gave sufficiently acidic carboxylic group capable to coordinate 1,1,3,3-tetramethylguanidine (TMG) and thus, allow good dispersion of Pd nanoparticles. The catalyst

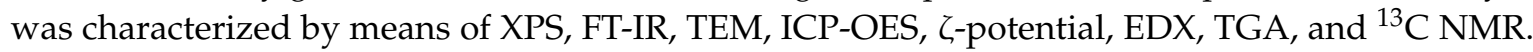
The release and catch mechanism was observed, whereas Pd re-deposition can be hindered by catalyst poisoning and eventual loss of palladium.
\end{abstract}

Keywords: supported ionic liquid catalyst (SILCA); palladium; Heck reaction; catalyst screening; optimization

\section{Introduction}

Upon production of fine chemicals the palladium catalyzed coupling reactions are well known and appreciated for their capability to yield versatile intermediates with preserved functionalities [1]. Among various kinds of reactions, the Heck reaction is one of the most important ones and is used for the production of intermediates for advanced synthesis of pharmaceuticals. This reaction reported by both Heck and Mizoroki [2,3] couples olefins and acrylates with molecules possessing suitable leaving groups such as aryl or vinyl (allyl) halides, triflates, acetates, etc. Next to the transition metal catalyst, it requires a base and it benefits from high temperatures. Nowadays, the reaction is commercially applied in the production of herbicides, sunscreen agents, coatings of electronic compounds, antiasthma agents, and various drugs [4].

Initially, Heck reactions were catalyzed by homogenous palladium, but the major problem of such a process is the catalyst instability which limits the number of turnovers that can be reached with a single 
charge of catalysts. Due to the steady increase of the palladium price, there is a pressure on researchers to come up with recoverable catalysts. The key issue is to stabilize palladium nanoparticles, which can be achieved by introducing ligands, although, however, they can hinder the product separation and increase the total costs because the price of the tailored homogeneous ligands can be high (5000-20,000 euro/kg for chiral bisphosphine ligands) [5]. Additionally, product contamination can be an issue since the maximum limit of residual $\mathrm{Pd}$ acceptable in the products of pharmaceutical industry ranges from $0.5 \mathrm{ppm}$ (parenteral) to $5 \mathrm{ppm}$ (oral) [6]. In order to avoid expensive nanofiltration membranes or column chromatography separations (extensive and usually destructive), the emission of metal and presence in the final product should be controlled already within the chemical synthesis step.

The scientific community is now turning towards the heterogeneously catalyzed Heck reactions and avoids the use of homogeneous ligands. However, despite all the benefits that a heterogeneous catalyst can bring into the system, the major drawbacks are the price of the catalyst in correlation with the synthesis route, the lower activity and selectivity compared to homogeneous catalysts, and in some cases, the contamination of the products with leached metal. At the same time, this is a reason why a limited number of heterogeneous catalysts are used for industrial production of fine chemicals and pharmaceuticals. However, there is a lot of interest to develop inexpensive, robust, and highly active heterogeneous catalysts.

The mechanism of heterogeneously catalyzed Heck reaction is still a controversial issue. As it seems for now, the reaction path can follow a real heterogeneous route [7-10] or "so to say" pseudo-heterogeneous route where the "catalyst" is actually a precursor or reservoir for active spices often discussed as a "release and catch" mechanism or boomerang catalysis [11-14]. In the case of the pseudo-heterogeneous route, palladium is dissolved from the carrier surface under reaction conditions (mainly in the presence of an aryl halide) thus forming highly active coordinatively unsaturated Pd species that takes a part in the reaction and upon the completion redeposited back on the surface. Next to it, under the release and catch concept there were also observations of the catalysts with non-covalently immobilized catalytic moiety on the suitable support. In these cases, the whole catalytic moiety, i.e., Pd-ligand couple was identified as active spices that was leached and recaptured back on the surface at the end of the reaction [15].

Unfortunately, many truly heterogeneous studies overlook the nature of the palladium entering the reaction cycle without monitoring the reaction heterogeneity with, for example, hot filtrate tests, mercury poisoning tests, poly(vinylpyridine) polymer (PVPy) tests [16] or sometimes without appropriate characterization of used catalysts. Other reasons for not taking into account the real nature of the catalytically active species is connected to the difficulties in studying it. Operando investigations of Heck reaction requires advanced characterization techniques. Only few studies quantitatively investigate palladium cluster formation and $\mathrm{Pd}^{2+}$ reduction with in situ UV-visible spectroscopy [17]. Meanwhile, leaching of the palladium under reaction conditions can be monitored by quick scanning extended X-ray absorption fine structure (QEXAFS) studies, even in the sub second time scale, thus giving a deep insight into the reaction mechanism [18]. In addition to the point, if the researcher is studying the mechanism or heterogeneity, it is important to distinguish which one of these two mechanisms is actually prevailing before going deeper into the catalyst or process design. Obviously, it would be devastating to use a pseudo-heterogeneous catalyst in a continuous flow reactor.

Throughout the years, N-heterocyclic carbenes have appeared as great ligands in coordination chemistry due to their strong $\sigma$-donor and $\pi$-acceptor characteristics $[19,20]$. In palladium catalyzed reactions they have been proposed as a replacement for phosphine ligands as being non-toxic, less expensive, and with a higher thermal stability [21-24]. In the chemistry of Heck reactions they have been studied in their heterogenized forms as molecules anchored on the surface of the carrier [16] and more lately as supported ionic liquids [25-27] to give what is today called a supported ionic liquid catalyst (SILCA).

In our previous work, we developed a catalyst that operates through the release and catch mechanism and utilizes $\mathrm{N}$-heterocyclic carbene, $\mathrm{CO}$, and tetramethylguanidine ligands in an ionic 
liquid form [28]. The obtained results encouraged us to continue in the same direction and here we present a set of new catalysts derived from the first one. The ionic liquid layer was optimized to meet the requirements in terms of the balance between the catalyst activity and reusability in the Heck reaction of iodobenzene and butyl acrylate. The screening resulted in a $\mathrm{PdCl}_{2}$ based catalyst with propyl imidazolium bromide as a first layer and tetramethylguanidinium pentanoate as a second (external) layer, which was stable in four successive experiments with a turnover frequency of $1660 \mathrm{~h}^{-1}$ at the temperature of $100{ }^{\circ} \mathrm{C}$. The catalysts structures were studied and characterized with relevant

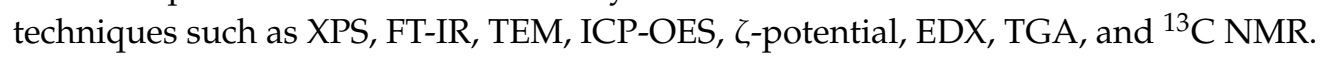

\section{Results and Discussion}

The new group of catalysts which were synthesized, characterized, and tested in the Heck reaction are discussed. These new catalysts were synthesized based on the modification of the previously reported procedure developed by our group [28] with a detailed experimental optimization of the ionic liquid layer structure in order to gain a higher activity and better stability in the Heck reaction. Smart adjusting of the ionic liquid layers was commenced in order to make a heterogenized ligand system capable of stabilizing the palladium nanoparticles. The anticipated structures of the synthesized SILCAs are displayed in Table 1.

Therefore, the obtained catalysts were tested for the Heck reaction of iodobenzene and butyl acrylate at $100{ }^{\circ} \mathrm{C}$, displayed in Figure 1 . On the basis of our recent studies $N$-Methyl-2-pyrrolidone was used as a solvent, triethanolamine as the base, and catalyst loading was $0.09 \mathrm{~mol} \% \mathrm{of} P d$ [28]. The reaction was performed until completion and next to the main product-butyl cinnamate, no other products were detected. Not too high reaction temperature was chosen in order to keep the palladium cluster size as low as possible since this affects the amount of palladium that actually enrolls in the catalytic cycle. In order to compare the catalysts, the activity of the fresh catalyst in the first reaction cycle was calculated (shown as a turnover frequency, TOF) and the stability was taken into consideration, too. The stability was evaluated on the basis of the number of the reaction cycles that the catalysts were capable to catalyze until completion with no need for prolonged reaction time compared to that for the fresh catalyst.

It was noticeable that the reaction is strongly dependent on the reaction conditions, so any deviations in temperature and catalysts amount can result in a prolonged reaction time. This imposed difficulties when recycling the catalysts. Since very small amounts of material were handled here (approx. $10 \mathrm{mg}$ ), even loss of just $1 \mathrm{mg}$ of catalyst between the cycles can have a significant impact on the outcome of the next experiment. Thus, recyclability tests were done multiple times (3-6) and the average (rounded) values are reported.

The reaction itself happens in the liquid phase while auxiliaries on the $\mathrm{SiO}_{2}$ have a role to stabilize the palladium re-taken at the end of the reaction cycle. In the presence of aryl halide, the palladium leaches and enters the cycle through the oxidative addition step and up on halide consumption it is redeposited back on the carrier $[13,29,30]$. Otherwise, in the absence of supported ionic liquid or any other ligand-like molecule, there is a serious threat that the palladium can form an inactive palladium back and precipitate. Therefore, the adequate choice of the moiety will make the support capable of efficiently releasing and catching the palladium. 
Table 1. Schematic presentation of assumed structures of synthetized supported ionic liquid catalysts (SILCAs) with variable auxiliaries.

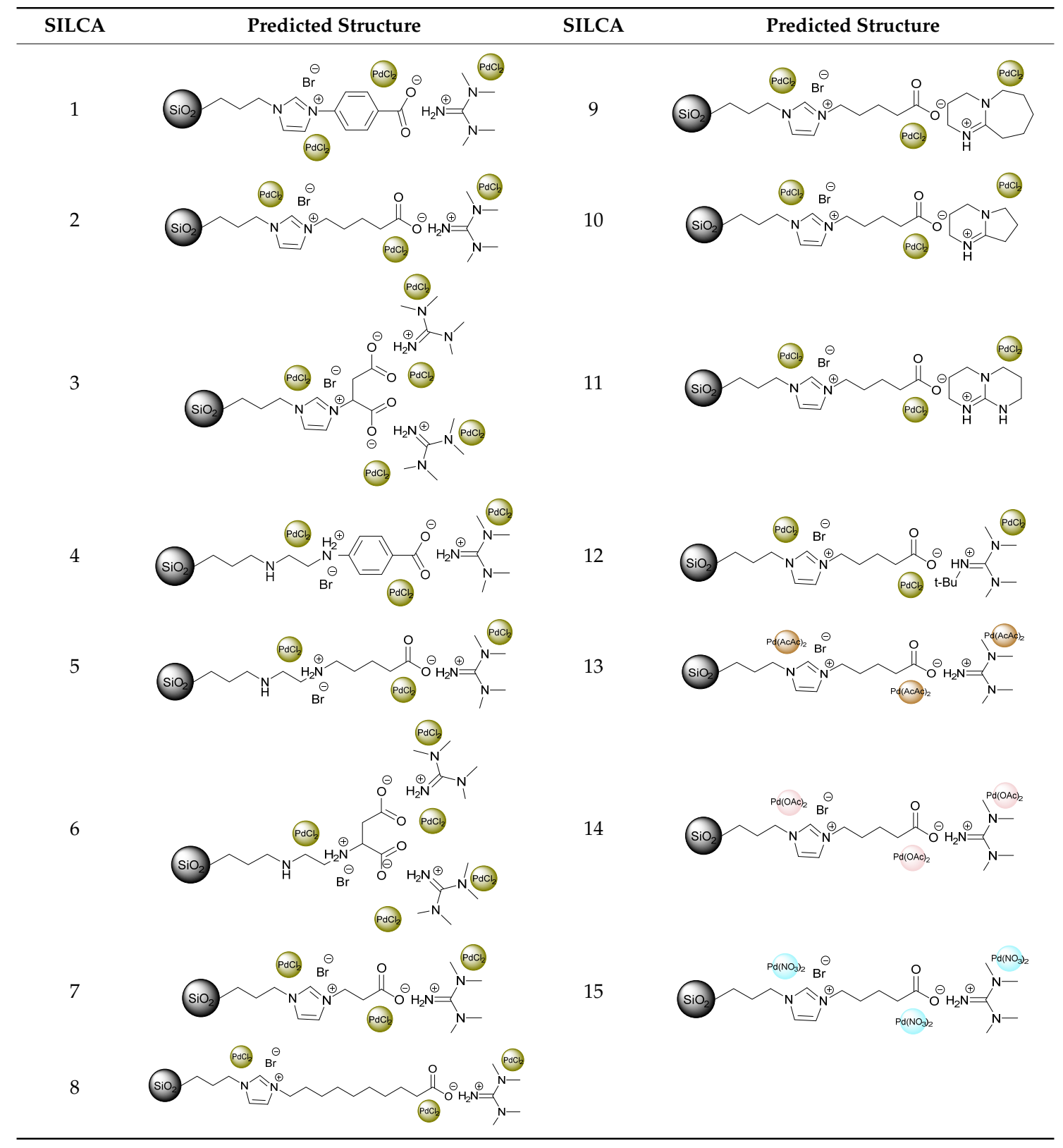<smiles>C=CC(=O)OCCC[CH+]Cc1cccc(/C=C/C(=O)OCCC[CH+]c2ccccc2)c1</smiles>

Figure 1. Heck reaction of iodobenzene and butyl acrylate in the presence of triethanolamine and different SILCAs, used to evaluate catalyst performance.

In the first set of experiments, catalysts with different acidic heads were compared (SILCAs 1-3, Figure 2). In terms of activity and stability, SILCA 2 gave the best results leading to reaction completion in $40 \mathrm{~min}$ and enabling the reuse of the catalyst in four consecutive cycles under identical reaction conditions. In the case of SILCA 1 and SILCA 3, a prolonged reaction time was needed (90 and $50 \mathrm{~min}$ ) and the stability of the catalyst decreased to approximately two and three reaction cycles. To gain a deeper understanding of these reaction outcomes, the catalysts were characterized with different 
techniques, of which the most valuable analytics were FT-IR, XPS, TEM, and $\zeta$-potential measurements. Firstly, by means of FT-IR and XPS, the surface composition was overviewed. The obtained FT-IR scans are shown in Figure 3. Comparing the scans of the first three catalysts (Figure 3a, SILCAs 1-3), small differences were observed in the peak at $1565 \mathrm{~cm}^{-1}$ which was assigned to $\mathrm{C}=\mathrm{N}$ vibrations of the imidazole ring and/or TMG. This peak was most evident in the case of SILCA 2 and a potential hint of the rigidity of this $\mathrm{C}=\mathrm{N}$ bond during the catalyst preparation. Unfortunately, the FT-IR spectra can just indicate the presence of various bonds and groups in a sample, but they cannot give information on how these bonds are arranged on the surface. Therefore, for the additional information on the surface composition, FT-IR was coupled with XPS. The XPS helped verify promptly if the real surface composition correspond to the expected composition, i.e., those presented in Table 1. The obtained results are presented in Figure 4 as atomic concentrations of $C, N$, and $\mathrm{Pd}$ at the surface for certain samples (Figure 4a) and percent amount of different chemical states fitted for C 1s, N 1s, and Pd 3d photoelectron lines (Figure $4 b-d$ ) whereas all the peak components are assigned to relevant functional groups on the basis of the literature data [31,32]. The presence of $-\mathrm{CF}_{2}$ was noticeable in all the samples that were in contact with Teflon lined magnetic bars in DMF at high temperature, during the course of catalyst synthesis. When glass lined magnetic bars were used instead, there was no fluorine contamination (Figure 4b, SILCAs 9-12). However, it complicated the synthesis much more due to fragility of the glass bars. Due to the fluorocarbon inertness, no significant attention was given to this kind of contamination.

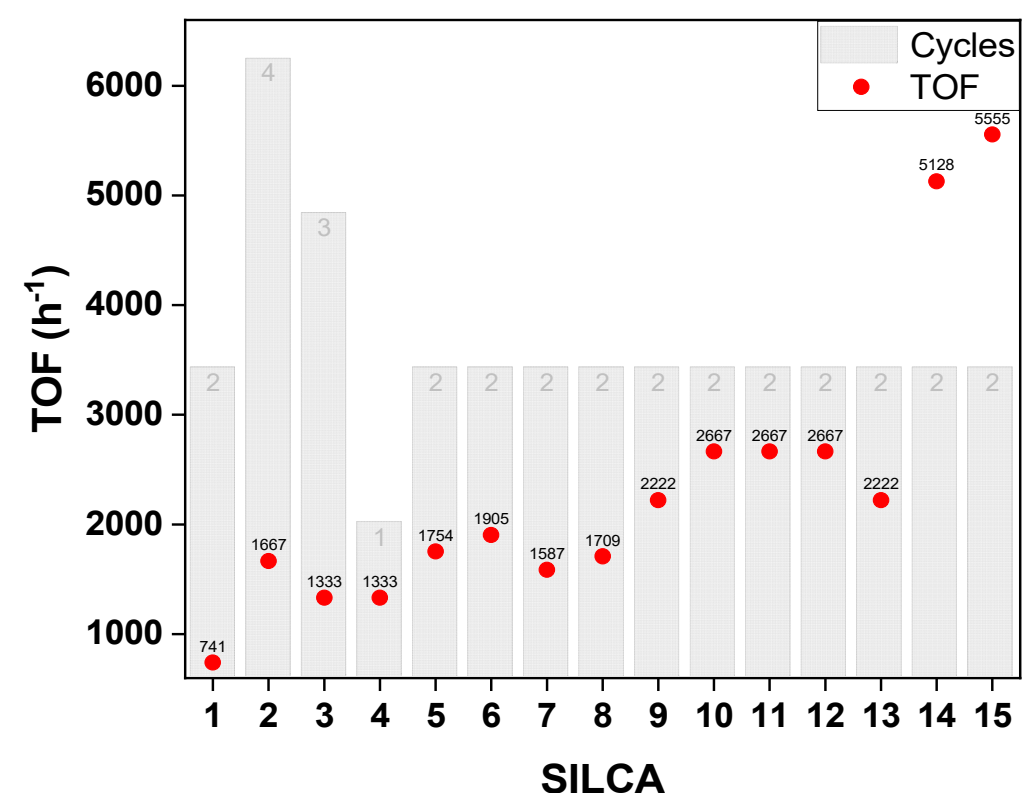

Figure 2. Catalytic performances of the SILCAs with different auxiliaries and palladium sources. Activity presented as a turnover frequency (TOF, $\mathrm{h}^{-1}$ ) calculated at full conversion. Recyclability presented as rounded average numbers of obtained reaction cycles.

Comparing the XPS of SILCA 1-3, the first evident difference was in the nitrogen group content (Figure 4c). For SILCAs 1 and 2, the peaks assigned to amino group and quaternary ammonium cation of imidazole and/or tetramethylguanidine species were found, and thus, indicating on the ionic nature of the layer (see details in Supplementary Materials). These groups were absent in the spectra of SILCA 3 , and instead, amide $\mathrm{C}=\mathrm{O}$ group was observed. This was resulting from the deterioration of imidazole ring by bromosuccinate, absence of TMG species, and mostly leaching of the nitrogen compounds during the catalyst synthesis, as is also evident when looking at Figure 4a. This means that the obtained structure does not correspond to the expected structure illustrated in Table 1. Interestingly, this catalyst still turned out to be active in the test reaction for three consecutive experiments (Figure 2). The main difference between SILCA 1 and more active and stable SILCA 2 was the amount of acidic groups, the 
latter one having a higher amount of them: $2 \%$ vs. $5 \%$. It was assumed that the main function of the $\mathrm{O}-\mathrm{C}=\mathrm{O}$ heads was to coordinate and keep the TMG cation which has an important role in terms of stabilizing and recapturing palladium nanoparticles [28]. Indeed, SILCA 2 turned out to not lose the activity in four consecutive cycles.

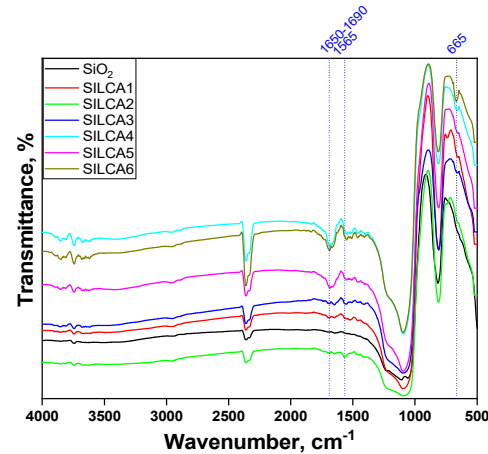

(a)

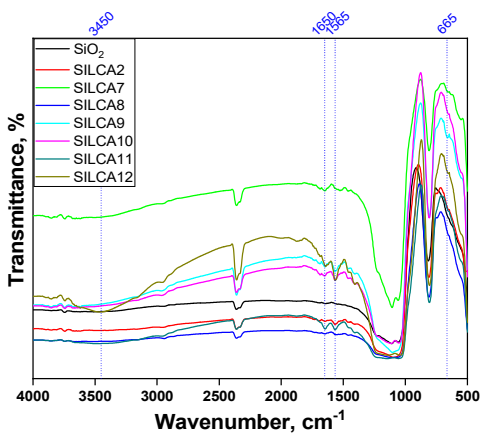

(b)

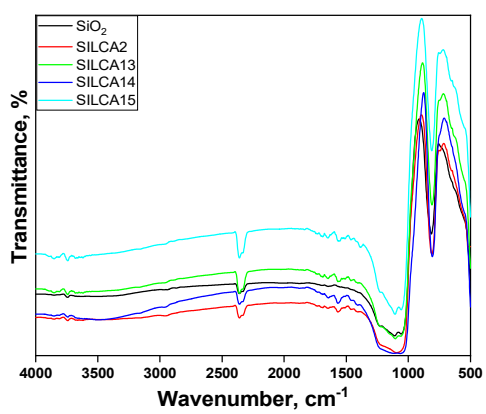

(c)

Figure 3. FT-IR spectra of reference materials and synthesized SILCAs 1-6 (a), SILCAs 7-12 (b) and SILCAs 13-15 (c).

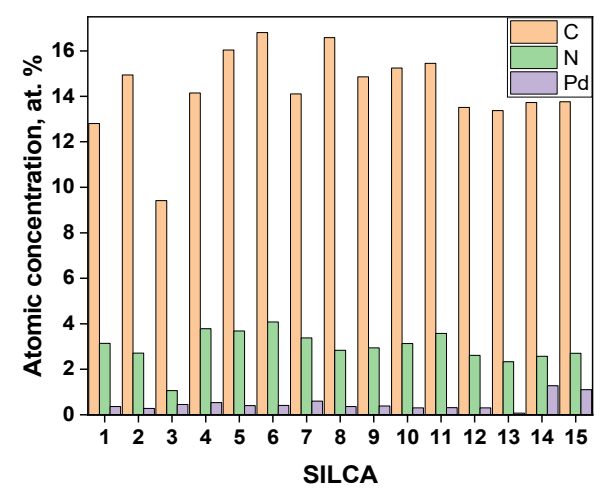

(a)

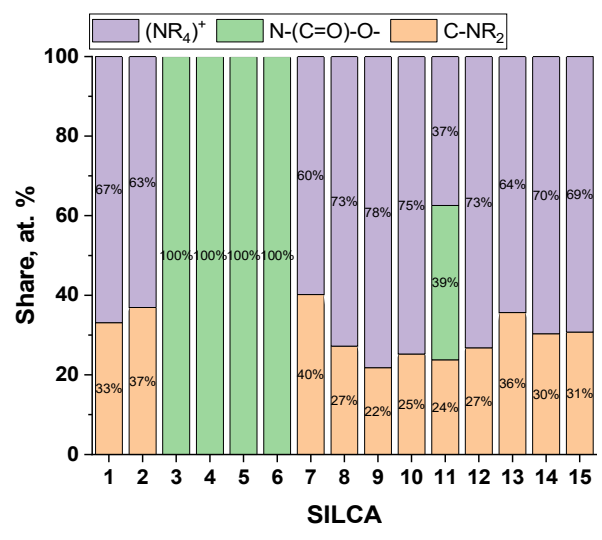

(c)

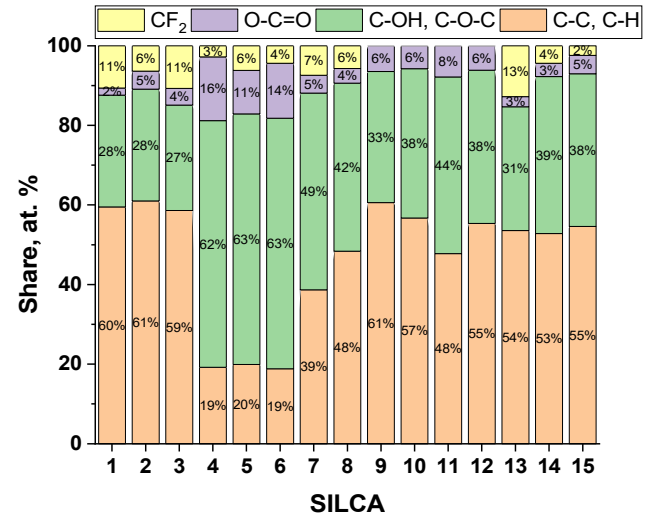

(b)

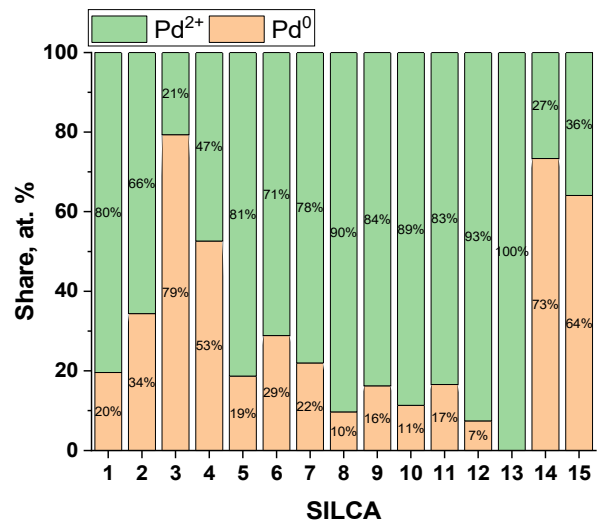

(d)

Figure 4. XPS data for synthesized catalysts. (a) Atomic concentrations of C, N, and Pd; (b) percent fractions of different carbon chemical states obtained by $\mathrm{C}$ 1s line fitting; (c) percent fractions of different nitrogen chemical states obtained by $\mathrm{N}$ 1s line fitting; (d) percent fractions of different palladium chemical states (after first $5 \mathrm{~min}$ of irradiation) obtained by $\mathrm{Pd} 3 \mathrm{~d}$ line fitting. 
$\zeta$-potential measurements were carried out in the absence of more sophisticated techniques which could confirm the basicity of the modified surface that is expected to be in correlation with the stabilization of the Pd nanoparticles. Temperature programmed desorption (TPD) of $\mathrm{CO}_{2}$ is generally used to estimate the basicity of catalyst surface; however, this should not be used for supported ionic liquids due to potential $\mathrm{CO}_{2}$ absorption and the limited thermal stability of the material (in this case $250{ }^{\circ} \mathrm{C}$ - detected by TGA). The isoelectric point (IEP) derived from $\zeta$-potential measurements indicates the global basicity or acidity of the molecule [33]. The molecules with a higher value of IEP can be considered more basic and more negative. Applied on heterogeneous catalytic systems, the more negative charged the surface is, the more firm the $\mathrm{Pd}^{2+}$ adhesion would be and, thus, the catalyst would have a better recyclability [34] and on the contrary, the more positively charged the surface is, the faster is the leaching of the cations and the reaction rate. In this context, it should be noted that if the $\mathrm{pH}$ value of the solution is less than the IEP, the surface charge is positive, if it is higher, then it is negative and capable of adsorbing cations. Most cross-coupling reactions are conducted under basic conditions at $\mathrm{pH}$ around 10, which in turn, is in most of the cases above the IEPs [10].

The IEP obtained from $\zeta$ - potential measurements of 12 catalysts (see Supplementary Materials) and with different surface auxiliaries are presented in Figure 5. The results show that among the tested catalysts, SILCA 2 gives the highest value of isoelectric point $\sim 6.9 \mathrm{pH}$, and potentially the strongest basicity and capability to stabilize palladium, and at the same time, that those with lower value of IEP are more active in the reaction (probably due to the faster Pd leaching) such as in the case of SILCAs 9-12. However, since $\zeta$ - potential measurements were conducted in an aqueous solution of the catalyst, they should be considered with skepticism due to the possibility of TMG hydrolysis [35]. Other potential important insight gained by this analysis is the stability of the materials, namely, for many of the catalysts two additional isoelectric points were detected, above $6 \mathrm{pH}$ (IEP2) and around $7 \mathrm{pH}$ (IEP3), as well as lack of IEP in the case of SILCAs 7 and 8. The appearance of more than one isoelectric point can imply on leaching and instability of the analyzed materials under analysis conditions [36]. This could mean that these modifications are unstable under the reaction conditions $(\mathrm{pH}>7)$ which can cause Pd leaching and catalyst deactivation. The obtained results reflected the difficulties in correlating the catalyst features with their structures and in predicting the catalyst performance.

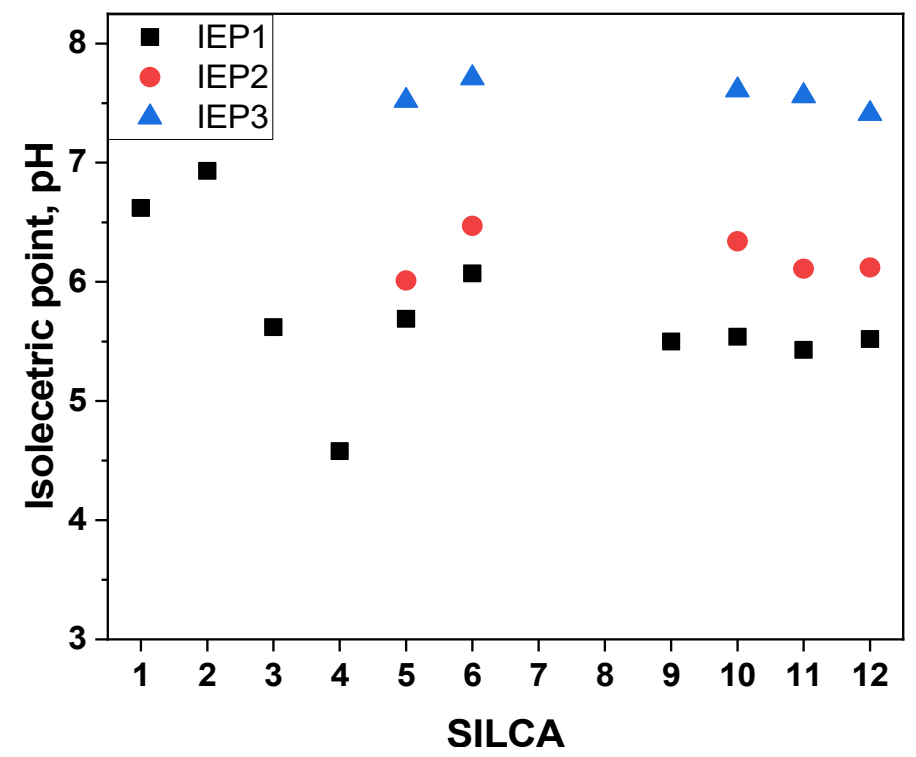

Figure 5. The values of isoelectric points (IEPs) for SILCAs 1-12 obtained with zeta potential measurements in water solution, at room temperature measured in the $\mathrm{pH}$ range from 2-8.

The FT-IR analysis of SILCAs 4-6 whereupon the imidazole modification was exchanged with aminoethylamino group, showed the main differences in the peaks at $1650-1690 \mathrm{~cm}^{-1}$ which is assumed 
to result from the contribution of amide $\mathrm{C}=\mathrm{O}$ and acidic $\mathrm{C}=\mathrm{O}$, alongside with the difference in the peak at $665 \mathrm{~cm}^{-1}$ belonging to the N-H wagging of the secondary amine. Amide formation was also confirmed with XPS, showing that all the present nitrogen is within the $\mathrm{N}-(\mathrm{C}=\mathrm{O})$-O- group with no TMG coordinated. This disapproved the expected structures in Table 1 indicating that neither succinic acid, nor aminoethylamino group can form ionic liquid layer under the given conditions and further led to the continuation of the work with SILCA 2-like structures as the most stable and recyclable ones.

Considering the FT-IR spectra of the SILCAs 7 and 8, the catalysts with the different chain lengths compared to SILCA 2, a visible stronger peak intensity of SILCA 7 was detected. The peaks assigned to nitrogen bonds (at 665 and $1565 \mathrm{~cm}^{-1}$ ) and to $\mathrm{C}=\mathrm{O}$ bond at $1650 \mathrm{~cm}^{-1}$ are seen on SILCA 7 which has shorter $\mathrm{C}-\mathrm{C}$ chain length, and therefore, higher $\mathrm{N} / \mathrm{C}$ and $\mathrm{O} / \mathrm{C}$ ratio. The dominance of nitrogen related groups is also visible from XPS results, whereas the higher relative amount of $\mathrm{N}$ and lower amount of $\mathrm{C}$ is found in SILCA 7 or more precisely higher amount of $\mathrm{C}-\mathrm{NR}_{2}, \mathrm{C}-\mathrm{OH}(\mathrm{C}-\mathrm{O}-\mathrm{C})$, and lower amount of $\mathrm{C}-\mathrm{C}(\mathrm{C}-\mathrm{H})$ (see Figure $4 \mathrm{a}-\mathrm{c}$ ).

Shortening (SILCA 7) and prolonging (SILCA 8) the carbon chain length of SILCA 2 did not bring any significant improvement in the catalyst activity but affected negatively the catalyst recyclability (Figure 2)-for no obvious reason at this moment. Therefore, in the following work the original modification of SILCA 2 was maintained and different super bases were screened for coordination with the acidic head as shown in Table 1 for SILCAs 9-12.

Applying bicyclic amidines such as in the case of SILCAs 9 and 10 and guanidines for SILCA 11-12, respectively, brought slight differences in the FT-IR spectra. All the expected peaks were still present but more prominently, with the addition of a broad peak in the area of $3450 \mathrm{~cm}^{-1}$ which was assigned to $\mathrm{CO}-\mathrm{H}$ stretching of acidic head implying on inefficient proton migration to the superbase. In the case of SILCA 11 , the formation of amide $C=O$ group was detected at $1650 \mathrm{~cm}^{-1}$ and was confirmed with XPS analysis indicating partial deterioration of the anchored modification by triazabicyclodecene superbase. New changes did not bring any improvement in terms of the catalyst recyclability. The achieved TOF was in the range of $2200-2700 \mathrm{~h}^{-1}$ and the activity was preserved in two consecutive cycles. Therefore, in the final study for SILCAs 13-15, the different sources of palladium were investigated with SILCA 2 structures. Instead of $\mathrm{PdCl}_{2}$ the $\mathrm{Pd}(\mathrm{AcAc})_{2}, \mathrm{Pd}(\mathrm{OAc})_{2}$, and $\mathrm{Pd}\left(\mathrm{NO}_{3}\right)_{2}$ were utilized and analyzed. As expected, no significant differences were observed in the FT-IR spectra (Figure 3c). The main difference was found in the palladium amount of SILCA 13. Almost three times lower amount of palladium was impregnated in this case, which was firstly observed during the XPS analysis, and also confirmed with the ICP-OES analysis as tabulated in Table 2. The average amount of impregnated Pd was $10 \times 10^{-2} \mathrm{mmol} / \mathrm{g}$ while in the case of SILCA 13 this was just above $3 \times 10^{-2} \mathrm{mmol} / \mathrm{g}$. Therefore, compared to other catalysts, it can be considered as an unsuccessful impregnation. Tested in the Heck reaction, these catalysts did not bring any improvement of the stability although the activity was enhanced to $5100-5500 \mathrm{~h}^{-1}$ for SILCAs 14 and 15 , probably resulting from a more rapid leaching (releasing) of Pd from the surface.

Traditionally, XPS is used to identify the oxidation state of the metals. During acquisition of Pd 3d spectrum for the samples produced in this study, it was noticed that $\mathrm{Pd}$ is reduced under X-rays and low energy electrons during the XPS experiment (see Supplementary Materials). Therefore, for all the samples, five scans ( $5 \mathrm{~min}$ total) for Pd $3 \mathrm{~d}$ line at the beginning of XPS measurements were included (even before survey spectrum) to gain an "original" $\mathrm{Pd} 3 \mathrm{~d}$ spectrum. The obtained results confirmed the presence of $\mathrm{Pd}^{2+}$ and $\mathrm{Pd}^{0}$ on the surface of the catalyst, with the exception of SILCA 13, where just non-metallic $\mathrm{Pd}^{2+}$ was found (Figure $4 \mathrm{~d}$ ). However, the amount of $\mathrm{Pd}^{0}$ given in Figure $4 \mathrm{~d}$ can be overestimated due to the possible formation of metallic $\mathrm{Pd}$ even during first $5 \mathrm{~min}$ of acquisition. 
Table 2. Palladium amount in synthesized SILCAs detected by ICP-OES analyses and comments for the TEM images of fresh catalysts.

\begin{tabular}{|c|c|c|}
\hline SILCA & $\mathrm{Pd}, \times 10^{-2} \mathrm{mmol} / \mathrm{g}$ & TEM Observation \\
\hline 1 & 9.3 & Low coverage of $\mathrm{Pd}$, irregularly scattered with few big agglomerates \\
\hline 2 & 8.1 & Good coverage of regularly dispersed nanoparticles of $8 \mathrm{~nm}$ average size \\
\hline 3 & 8.6 & Good coverage, with raspberry-like agglomerates of average size $27 \mathrm{~nm}$ \\
\hline 4 & 12.7 & Good coverage, with mostly individual nanoparticles of average size $13 \mathrm{~nm}$ \\
\hline 5 & 11.2 & Low coverage, particles of irregular size and shape (round, qubic, triangle) \\
\hline 6 & 8.4 & Medium coverage with nanoparticles of $8.5 \mathrm{~nm}$ average size \\
\hline 7 & 9.7 & Few agglomerates of Pd, with no visible individual nanoparticles \\
\hline 8 & 11.4 & Very few agglomerates \\
\hline 9 & 10.8 & Very few big agglomerates \\
\hline 10 & 11.7 & Very few agglomerated big nanoparticles \\
\hline 11 & 11.1 & Almost none of Pd, few big nanoparticles \\
\hline 12 & 10.6 & Very few big individual nanoparticles, with few big agglomerates \\
\hline 13 & 3.2 & Almost none of Pd, just few nanoparticles \\
\hline 14 & 9.0 & Densely dispersed nanoparticles of $7.5 \mathrm{~nm}$ average size \\
\hline 15 & 8.1 & Good coverage of grape-like agglomerated nanoparticles \\
\hline
\end{tabular}

Visual inspection of the materials was possible with TEM analysis that provided an overall overview of the catalysts and gave the insight on how the structure of the layer affects the palladium size and disposition. The obtained images (see Supplementary Materials) are commented in Table 2. The TEM results are in relatively good agreement with the XPS analysis, i.e., the samples that initially had more metallic palladium $\left(\mathrm{Pd}^{0}\right)$ showed more visible nanoparticles and oppositely, those which had a higher percentage of $\mathrm{Pd}^{2+}$ (e.g., SILCAs 7-13) were hard to identify. TEM images of the most relevant catalyst-SILCA 2, being the most recyclable, are presented in Figure 6. High dispersion of the nanoparticles was observed with this catalyst, and it is assumed that the stability of this catalyst lies in the capability of the anchored layer to stabilize nanoparticles and avoid overgrowth of nanoparticles and agglomeration.

Finally, the structure of this catalyst was confirmed with energy-dispersive X-ray spectroscopy (EDX) and carbon nuclear magnet resonance (NMR). The EDX spectrum of the catalyst enabled elemental analysis and verified the presence of bromine in the ionic liquid layer. The carbon peak and its atomic percentage appears to be much higher than it is, in reality, because of the analytic shortcomings, i.e., the usage of carbon-tape to deposit the catalyst prior to exposing it to the beam. This means that the actual atomic percentage of other elements is much higher than it is presented in Figure 7. Nevertheless, a good overview of the material was obtained.

${ }^{13} \mathrm{C}$ NMR analysis of fresh and spent catalyst confirmed the layer structure and stability. The most evident peaks were those denoted to the carbons of propyl chain of modified surface at 7.3, 21.6, and $49.0 \mathrm{ppm}$ (Figure 8), as well as the carbon atoms of imidazolium ring which were found at 121.0 and $134.5 \mathrm{ppm}[26,28]$. Aliphatic carbons originating from anchored acid resonated in the range of 21.6 to 34.6 ppm being partially overlapped with the peak of carbon number 2 [25], followed by ending the carboxylic group with a peak at $178.1 \mathrm{ppm}$. Tetramethylguanidinium contributed with two small peaks, the first one at $38.9 \mathrm{ppm}$ belonging to methyl groups that falls in the region of aliphatic carbons and the peak at $172.7 \mathrm{ppm}$ of imine carbon that is slightly shifted downfield, most probably due to the higher polarization of the ionic version of TMG compared to the molecular one [37]. The NMR analysis of the recovered catalyst after the use in one reaction cycle (Figure 8, red) showed the stability of the attached structure under the reaction conditions. All the carbon peaks were still present with an additional peak at $55.6 \mathrm{ppm}$ that was assumed to belong to residual molecules of solvents and base, or more precisely NMP related structures and/or $(\mathrm{EtOH})_{3} \mathrm{NH}^{+} \mathrm{I}^{-}$residual salts [37]. This indicates the potential of catalyst deactivation due to poisoning, since the adsorbed species can block the sites capable to stabilize Pd nanoparticles, therefore resulting in their leaching. 

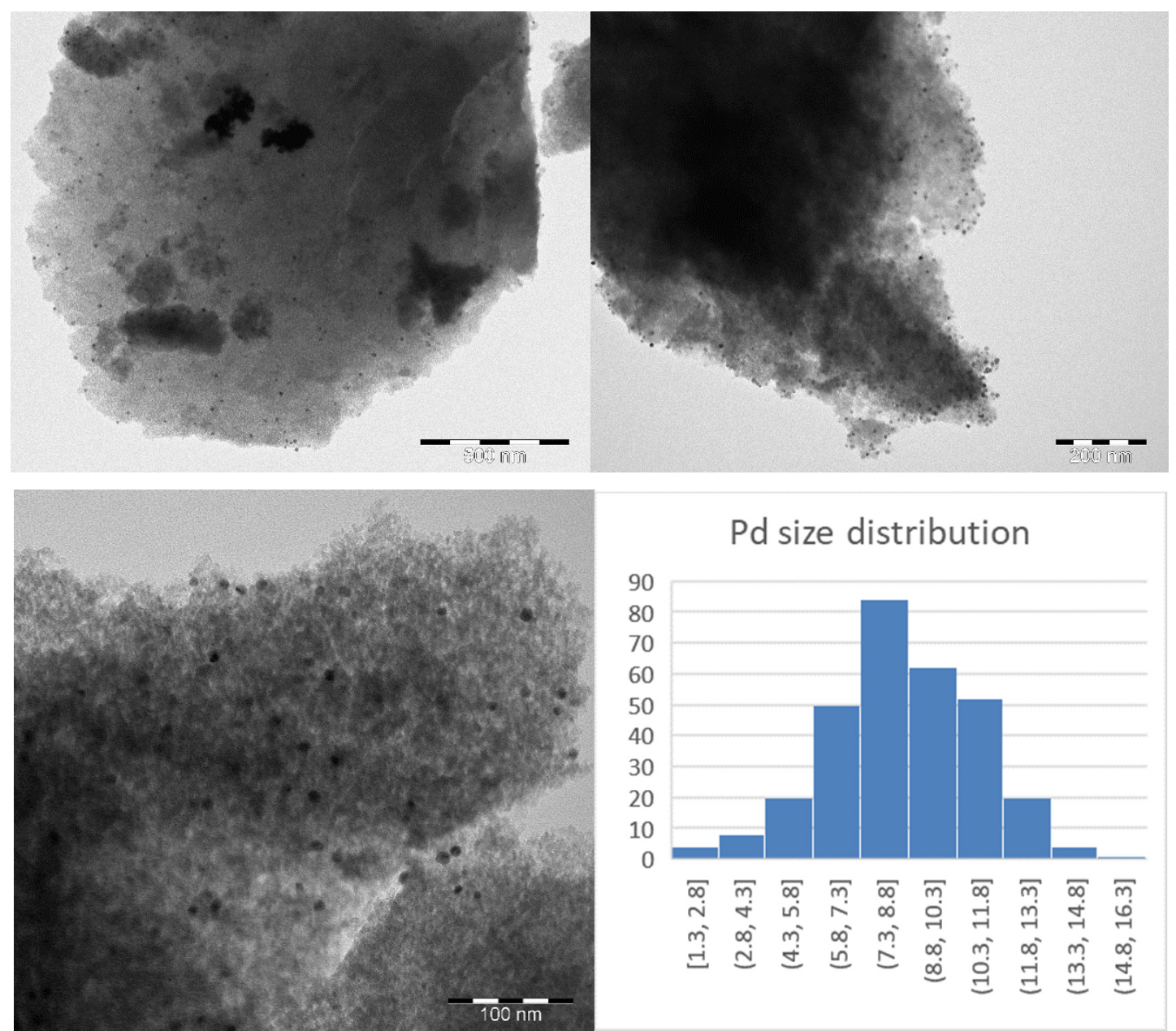

Figure 6. TEM images of SILCA 2 of a different scale and palladium size distribution.

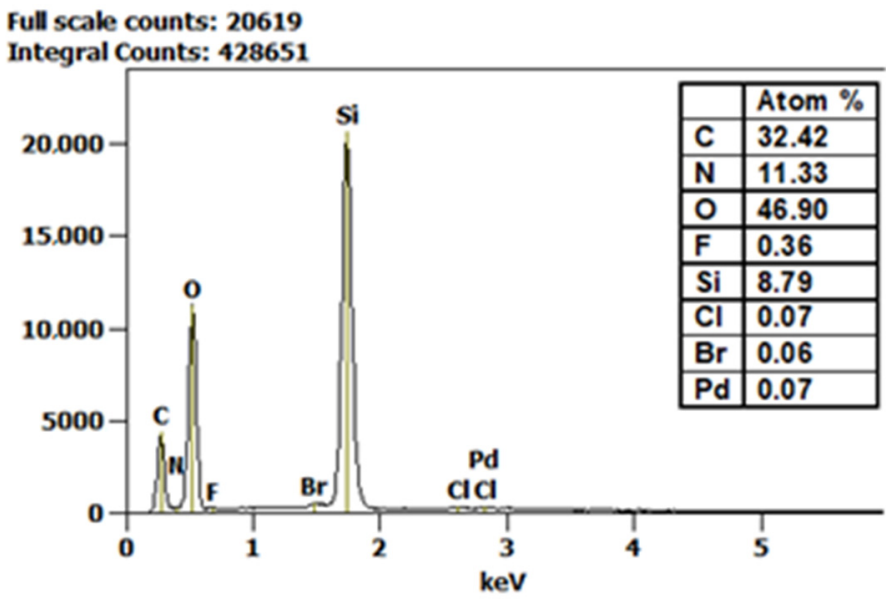

Figure 7. EDX spectrum of SILCA 2. 


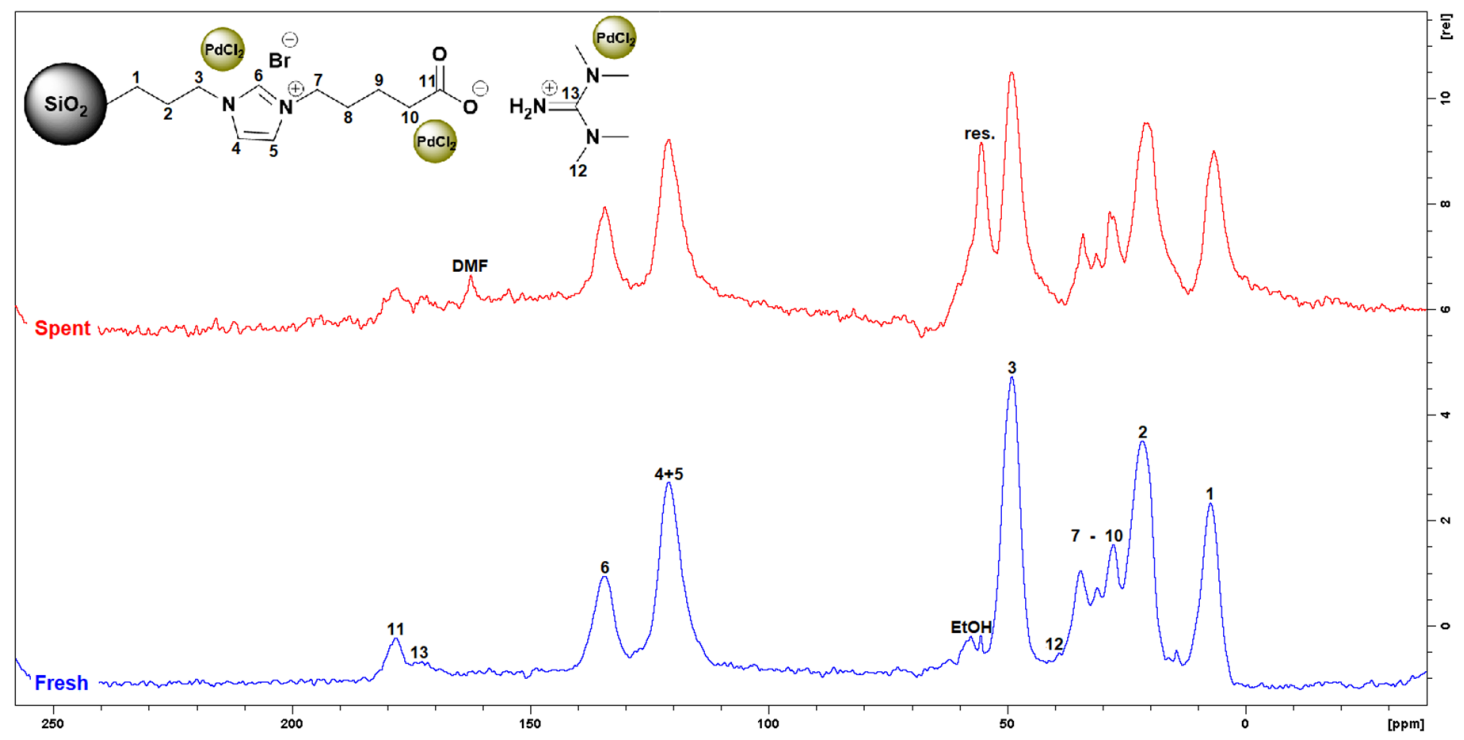

Figure 8. ${ }^{13} \mathrm{C}$ NMR spectrum of fresh and spent SILCA 2.

Thermal stability of the catalyst was analyzed with thermal gravimetric analysis (TGA). As shown in Figure 9, the ionic liquid layer is stable up to $250{ }^{\circ} \mathrm{C}$, after which thermal decomposition of organic moieties takes place. A small mass drop of approximately $0.5 \mathrm{wt} \%$ at the temperatures under $250{ }^{\circ} \mathrm{C}$ was considered to belong to adsorbed water and residual solvents, while at the temperatures above this, the mass drop of $6-7 \mathrm{wt} \%$, corresponds to the contribution of the ionic liquid layer. Therefore, at the reaction conditions, no loss of the ionic liquid layer should take place as a consequence of thermal destructions.

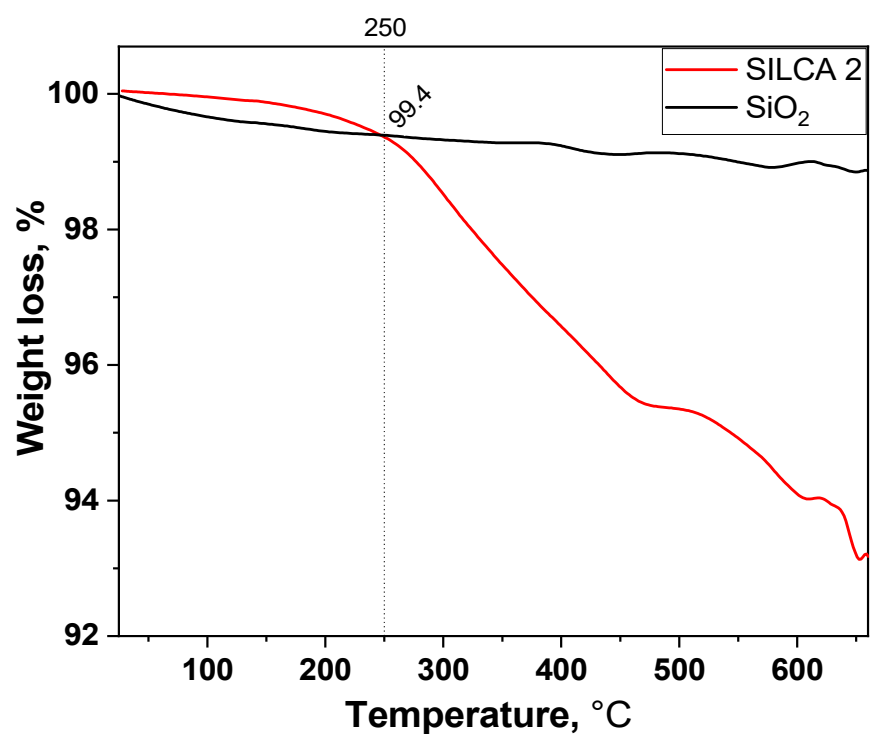

Figure 9. Thermal gravimetric analysis curve for pristine $\mathrm{SiO}_{2}$ and synthesized catalyst.

While monitoring the reaction progress via sampling in defined time intervals ( $5 \mathrm{~min}$ ), for all the catalysts, concentration curves resembling the one presented in Figure 10 for SILCA 2 were obtained. An initial induction time was observed, which in case of SILCA 2 was approximately 25 min after which the reaction ignited. To understand this induction time and to confirm the release and catch mechanism in the reaction with SILCA 2, the relative amount of leached Pd in slurry was monitored with ICP-OES during the course of the reaction and results are over-layered with concentration curves in Figure 10. The initial period corresponds to the start of slow emission of Pd from the surface, whereas the reaction 
completion corresponds to the highest amount of palladium in slurry. After the reaction completion (40 min), palladium needs $10 \mathrm{~min}$ more to redeposit back. Following the re-deposition dynamics, it was evident that for the efficient use of catalyst, the reaction plus re-deposition time should be no less than $50 \mathrm{~min}$, after what the catalyst can be successfully recycled. It was not possible to fully recover the leached palladium, after the $1^{\text {st }}$ reaction cycle approximately $6 \mathrm{ppm}$ of palladium was detected in the solvent, which corresponds to $10 \%$ of initially loaded Pd. Together with the catalyst poisoning this can be the reason for the decline of the catalyst activity.

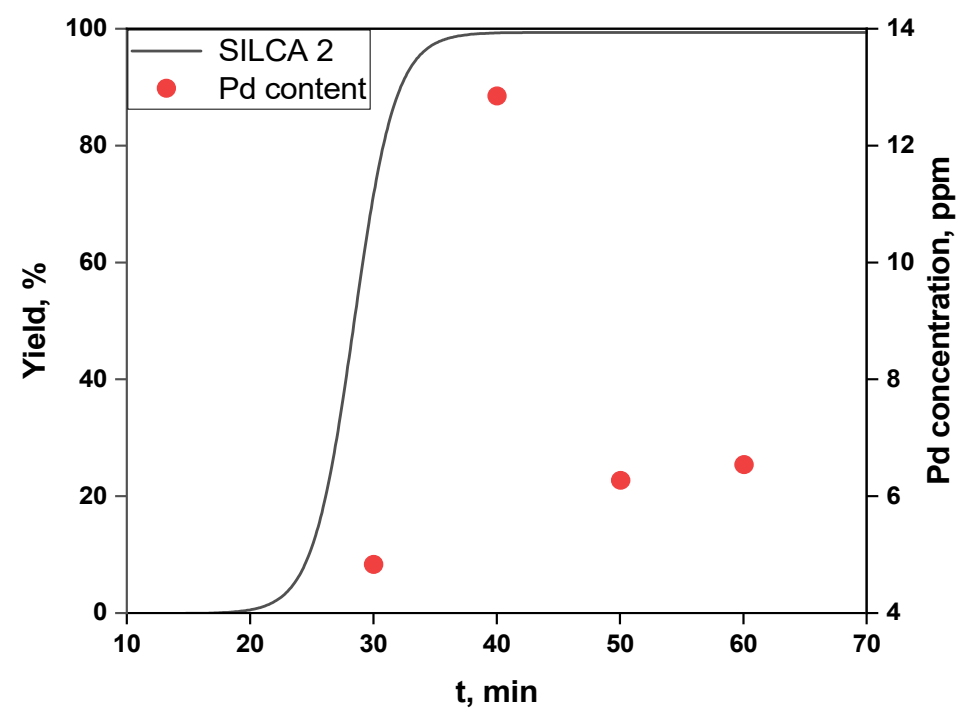

Figure 10. Progress of the reaction catalyzed by SILCA 2 correlated with Pd leaching.

\section{Materials and Methods}

\subsection{Chemicals and Analysis}

All the chemicals were of analytic grade purchased from commercial producers Sigma-Aldrich or Alfa Aesar and they were used as received.

The materials were characterized with different techniques. Fourier transform infrared spectroscopy (FT-IR) measurements of pelletized samples were performed on an Bruker IFS 66/S FT-IR (Bruker Optics, Ettlingen, Germany) in the $400-4000 \mathrm{~cm}^{-1}$ region.

X-ray photoelectron spectroscopy (XPS) was used to follow the changes in the oxidation state of palladium and the states of other elements. The spectra were collected with a Kratos Axis Ultra DLD electron spectrometer (Kratos Analytical Ltd., Manchester, UK) using a monochromated Al Ka source operated at $150 \mathrm{~W}$. Analyzer pass energy of $160 \mathrm{eV}$ was set for acquiring a wide spectra and a pass energy of $20 \mathrm{eV}$ was used for individual photoelectron lines. The surface potential was stabilized by the spectrometer charge neutralization system. The binding energy (BE) scale was referenced to the Si $2 p$ line of silica, set at $103.4 \mathrm{eV}$. Processing of the spectra was accomplished with the Kratos software. Due to the possible reduction of $\mathrm{Pd}^{2+}$ ions under $\mathrm{X}$-rays and low energy electrons during XPS measurements, Pd $3 d$ spectrum was acquired during first 5 min of exposure, and then again with other photoelectron lines.

The zeta potential $(\zeta)$ measurements were performed with a Zetasizer Nano ZS (Malvern Panalytical Ltd., Malvern, UK) using the light scattering technique, while for $\mathrm{pH}$ measurements the potentiometric method (MPT-2) was applied. The samples were made in deionized water and analyzed at ambient temperature with the use of $\mathrm{NaOH}$ and $\mathrm{HCl}$ as titrants in a $\mathrm{pH}$ range of 3-8. The final zeta potential vs. $\mathrm{pH}$ curve were averaged from three measurements and the isoelectric point was derived from it. 
The analysis of metal amount in the catalyst was carried out with inductively coupled plasma optical emission spectroscopy (ICP-OES) supplied with Optima 4300 DV optical atomic emission spectrometer (Perkin Elmer, Waltham, MA, USA). The transmission electron microscopy (TEM) was performed on a JEM 1400 plus (120 kV, $0.38 \mathrm{~nm}$ ) with OSIS Quemesa 11 Mpix digital camera (Jeol Ltd, Tokyo, Japan). The elemental analysis was performed with energy-dispersive X-ray spectroscopy (EDX) on a Zeiss Leo Gemini 1530 SEM (Zeiss Leo, Oberkochen, Germany) with a Thermo-NORAN vantage X-ray detector (Thermo Scientific, Madison, WI, USA). The thermo-gravimetric analysis (TGA) were made on CAHN D-200 instrument (Cahn Instruments, Inc., CA, USA) in the range of 20-700 ${ }^{\circ} \mathrm{C}$ with a heating rate of $10{ }^{\circ} \mathrm{C} \mathrm{min}^{-1}$ and under Ar atmosphere. The Solid-State ${ }^{13} \mathrm{C}$ nuclear magnetic resonance spectroscopy (CP MAS ${ }^{13} \mathrm{C}$ NMR) spectra were recorded on a Bruker AVANCE-III spectrometer (Bruker Scientific Instruments, Billerica, MA, USA) operating at $399.75 \mathrm{MHz}\left({ }^{1} \mathrm{H}\right)$ and $100.5 \mathrm{MHz}\left({ }^{13} \mathrm{C}\right)$ equipped with a $4 \mathrm{~mm}$ double resonance $(\mathrm{H} / \mathrm{X}) \mathrm{CP}$ MAS probe. The powdered sample was spun at a $14 \mathrm{kHz}$ spin rate. The proton $90^{\circ}$ high-power pulse of $2.9 \mathrm{~ms}$ and contact time of $2 \mathrm{~ms}$ was used. A total of 42,000 scans (fresh catalyst) and 200,000 scans (spent catalyst) were accumulated using a recovery delay of $2 \mathrm{~s}$. The solution state ${ }^{1} \mathrm{H}$ and ${ }^{13} \mathrm{C}$ NMR spectra were recorded at 300 and $298 \mathrm{~K}$ on a Bruker Avance-III HD $500 \mathrm{MHz}$ spectrometer (Bruker Scientific Instruments, Billerica, MA, USA) equipped with a Bruker SmartProbe ${ }^{\mathrm{TM}}$. For analysis the samples were dissolved in deuterated chloroform with $0.03 \%$ tetramethylsilane (TMS) as the internal standard.

The Heck reaction yield was determined with gas chromatography, GC-HP 6890 Series with HP-5, $5 \%$ phenyl methyl siloxane capillary column $(30.0 \mathrm{~m} \times 320 \mu \mathrm{m}, 0.25 \mu \mathrm{m})$ and a flame ionization detector (Agilent Technologies, Inc., Santa Clara, CA, USA) at the constant temperature of $300{ }^{\circ} \mathrm{C}$. The temperature of the injector was $280^{\circ} \mathrm{C}$ and gas flow was $9.5 \mathrm{~mL} \mathrm{~min}^{-1}$. Column heating from 40 to $250{ }^{\circ} \mathrm{C}$ was carried out with a $10^{\circ} \mathrm{C} \mathrm{min}^{-1}$ heating rate. Hexadecane was used as an internal standard.

\subsection{Heck Reaction Procedure and Catalyst Recovery}

The reaction was run in a flat bottom vial tube $(4.5 \mathrm{~mL})$ sealed with screw cap equipped with a Teflon ${ }^{\circledR}$ lined septum and a magnetic stirrer, under air atmosphere and heated by an isolated oil bath. In catalyst screening tests $1 \mathrm{mmol}$ of iodobenzene, $1.5 \mathrm{mmol}$ of butyl acrylate, $1.5 \mathrm{mmol}$ of triethanolamine, and $1 \mathrm{~mL}$ of $\mathrm{N}$-Methyl-2-pyrrolidone were placed in a reactor together with a catalyst amount corresponding to $0.09 \mathrm{~mol} \%$ of Pd (detected by ICP-OES). The temperature of the oil bath was kept at $100-105{ }^{\circ} \mathrm{C}$ with an assumption that this will result in a temperature of $100{ }^{\circ} \mathrm{C}$ in the reaction slurry, due to the small volume of the vials. Once the temperature reached the preset value, time measuring was initiated. In defined time intervals, samples were withdrawn with a GC syringe injector and the progress of the reaction was monitored with a GC. Ten $\mu \mathrm{L}$ of reaction mixtures were taken out from the reaction slurry and diluted with $240 \mu \mathrm{L}$ of DMF to give $4.0 \%$ dilution samples, followed by the addition of $250 \mu \mathrm{L}$ internal standard and final dissolution of $2.0 \%$. The as obtained samples were analyzed with GC and the reaction was stopped after completion. For catalyst leaching tests reaction was run in multiple vial tubes in parallel and in defined time intervals (10 min) samples for ICP-OES were taken out one by one from the vials. One $\mathrm{mL}$ of reaction slurry was withdrawn from the vial, filtered, dissolved in aqua regia, and stirred overnight prior to the analysis.

In the catalyst recyclability studies, the reaction was conducted in a flat bottom vial tube $(8.5 \mathrm{~mL})$ with a screw cap and magnetic stirrer, under air atmosphere, and heated by an isolated oil bath. Vials were submerged in the oil bath just above the level of liquid, while the temperature of the oil was kept at $105-110^{\circ} \mathrm{C}$ (slightly higher temperature due to the bigger vial volume) with the assumption that this will result in the temperature of $100{ }^{\circ} \mathrm{C}$ in the reaction slurry. Upon a predetermined time, the reaction was completed and the vials were cooled, and samples were withdrawn with a GC syringe injector and analyzed with a GC. The catalyst was washed and separated by adding approx. $7.0 \mathrm{~mL}$ of DMF (full vial) and centrifuging, followed by flushing with DMF $(8.5 \mathrm{~mL})$, diethyl ether $(8.5 \mathrm{~mL})$, and air drying for $30 \mathrm{~min}$. The catalysts were reused in the next cycle without removal from the vials or stored at ambient conditions for further characterization. 
Every experiment was repeated a minimum of three times and average values are reported.

\subsection{Catalyst Synthesis}

The new catalysts were synthesized via a modified procedure compared to that we reported recently [28]. Here, a more detailed synthesis route of the catalyst is presented with 3-propyl imidazolium bromide benzoic acid modification, 1,1,3,3-tetramethylguanidine (TMG) in the role of a cation, and $\mathrm{PdCl}_{2}$ as a source of the metal. Other catalysts were prepared accordingly and all anticipated structures are displayed in Table 1.

\subsubsection{Synthesis of $\mathrm{PdCl}_{2}$ Supported on 3-Propyl Imidazolium Bromide-Tetramethylguanidinium} Benzoate Modified $\mathrm{SiO}_{2}$ (SILCA 1)

Imidazole Modification

In order to modify the silica surface, silanol groups were firstly reacted with (3-chloropropyl)trimethoxysilane in refluxing toluene. Under nitrogen atmosphere, $1 \mathrm{~g}$ of calcined $\mathrm{SiO}_{2}$ was reacted with $5 \mathrm{mmol}$ of the modifying agent for $24 \mathrm{~h}$. The obtained material was filtrated, flushed with fresh toluene, and for $24 \mathrm{~h}$ washed with ethanol in a Soxhlet apparatus prior to drying.

Thereafter, propylated silica was reacted with imidazole. Finely crushed imidazole flakes $(10 \mathrm{mmol})$ were dissolved in toluene and mixed with $1 \mathrm{~g}$ of silica. The slurry was stirred for $24 \mathrm{~h}$ under nitrogen atmosphere under refluxing conditions. As before, the obtained material was flushed with fresh toluene and for $24 \mathrm{~h}$ washed with ethanol in a Soxhlet apparatus prior to drying.

\section{Acid Modification}

Prior to the further modification of silica, the acidic group of 4-bromobenzoic acid had to be protected and esterified [38]. The 4-bromobenzoic acid $(6 \mathrm{mmol})$ was dissolved in $20 \mathrm{~mL}$ of methanol and while stirred, a dropwise addition of $3 \mathrm{~mL}$ of $40 \% \mathrm{H}_{2} \mathrm{SO}_{4}$ took place. The mixture was heated to $50{ }^{\circ} \mathrm{C}$ and reacted for $12 \mathrm{~h}$. After workup, $10 \mathrm{~mL}$ of $\mathrm{H}_{2} \mathrm{O}$ was added and the acidic solution was neutralized with approximately $50 \mathrm{~mL}$ of brine. The product was isolated in dichloromethane and dried in a rotary evaporator. The obtained white crystalline methyl 4-bromobenzoate was stored in a cold refrigerator. See the Supplementary Materials for the NMR data.

The obtained esterified product $(1.5 \mathrm{mmol}, 0.3226 \mathrm{~g}$ ) was dissolved in DMF, followed by addition of $1 \mathrm{~g}$ modified silica. After reacting it for $24 \mathrm{~h}$ under refluxing conditions $\left(\mathrm{N}_{2}\right.$ atm), the slurry was cooled down and solid separated with centrifuge, washed with dichloromethane, ethanol, and then dried.

Finally, the recovery of the acidic group from ester modified $\mathrm{SiO}_{2}$ was carried out in methanol and catalyzed by a strong base. To the $1 \mathrm{~g}$ of modified silica solution, $1.25 \mathrm{~mL}$ of $\mathrm{NaOH}(1 \mathrm{M})$ was added and stirred for $12 \mathrm{~h}$ under reflux conditions. After that, $\mathrm{HCl}(1 \mathrm{M})$ was added to acidify the solution. The solid was separated and washed with water, ethanol, and dried.

\section{Base Modification and Palladium Loading}

In the next step, the acidified surface was coordinated with a proton acceptor (TMG). To the TMG ( $5 \mathrm{mmol}$ ) ethanol solution, $1 \mathrm{~g}$ of 3-propyl imidazolium bromide benzoic acid modified $\mathrm{SiO}_{2}$ was added and stirred for $6 \mathrm{~h}$. The product was centrifuged, washed with diethyl ether, and dried. Finally, this material was loaded with the metal. $\mathrm{PdCl}_{2}(0.1 \mathrm{mmol})$ was dissolved in methanol and mixed with $1 \mathrm{~g}$ of modified $\mathrm{SiO}_{2}$. After $12 \mathrm{~h}$ stirring at room temperature, the catalyst was separated and washed with ethanol and diethyl ether. The dry material was $\mathrm{PdCl}_{2}$ supported on 3-propyl imidazolium bromide-tetramethylguanidinium benzoate modified $\mathrm{SiO}_{2}$ or more briefly, SILCA 1 with the targeted structure presented in Table 1. 
3.3.2. Synthesis of $\mathrm{PdCl}_{2}$ Supported on 3-Propyl Imidazolium Bromide-Tetramethylguanidinium Pentanoate Modified $\mathrm{SiO}_{2}$ (SILCA 2)

SILCA 2 (see Table 1) was prepared in the same manner as SILCA 1 with a change in the acid that was used. Instead of 4-bromobenzoic acid, 5-bromo-pentanoic acid was used which preliminary gave methyl 5-bromo-pentanoate as a transparent light yellowish liquid.

3.3.3. Synthesis of $\mathrm{PdCl}_{2}$ Supported on 3-Propyl Imidazolium Bromide-Tetramethylguanidinium Succinate Modified $\mathrm{SiO}_{2}$ (SILCA 3)

SILCA 3 (see Table 1) was prepared analogously with SILCA 1 with a variation of the acid used. In the case of SILCA 3, bromosuccinic acid was used instead of 4-bromobenzoic acid and the reaction yielded a viscous transparent liquid dimethyl 2-bromosuccinate. In addition, in the case of SILCA 3, a double amount of TMG (10 mmol) was used due to the two acidic groups of bromosuccinic acid.

3.3.4. Synthesis of $\mathrm{PdCl}_{2}$ Supported on 3-(Aminoethylamino)Propyl Bromide-

Tetramethylguanidinium Benzoate (SILCA 4), Pentanoate (SILCA 5), and Succinate Modified $\mathrm{SiO}_{2}$ (SILCA 6)

SILCA 4, SILCA 5, and SILCA 6 (see Table 1) were prepared via slightly modified SILCA 1, SILCA 2 , and SILCA 3 synthesis routes. Instead of imidazole modification, the aminoethylamino group was utilized. To immobilize the modifying agent on the surface of the solid, $1 \mathrm{~g}$ of $\mathrm{SiO}_{2}$ was suspended in toluene and a dropwise addition of $5 \mathrm{mmol}$ of 3-(aminoethylamino)propyl trimethoxysilane was commenced. The mixture was stirred for $24 \mathrm{~h}$ under reflux conditions and $\mathrm{N}_{2}$ atmosphere. The product was separated by filtration, flushed with toluene, and for $24 \mathrm{~h}$ washed with ethanol in a Soxhlet apparatus prior to drying. Further synthesis was continued as already explained with esterified 4-bromobenzoic acid, 5-bromo-pentanoic acid, and bromosuccinic acid.

3.3.5. Synthesis of $\mathrm{PdCl}_{2}$ Supported on 3-Propyl Imidazolium Bromide-Tetramethylguanidinium Propionate modified $\mathrm{SiO}_{2}$ (SILCA 7) and 3-Propyl Imidazolium Bromide-Tetramethylguanidinium Decanoate Modified $\mathrm{SiO}_{2}$ (SILCA 8)

SILCA 7 and SILCA 8 (see Table 1) were prepared in a similar way as SILCA 1 with a change in acids that were used. For SILCA 7, instead of 4-bromobenzoic acid, 3-bromo-propionic acid was used, which gave the intermediate methyl 3-bromo-propionate as a white crystalline substance. Meanwhile, in the case of SILCA 8, 10-bromodecanoic acid was used which yielded a brownish liquid methyl 10-bromodecanoate.

3.3.6. Synthesis of $\mathrm{PdCl}_{2}$ Supported on 3-Propyl Imidazolium Bromide-Diazabicycloundecenium, -Diazabicyclononenium, -Triazabicyclodecenium, and -Tert-Butyl-Tetramethylguanidinium Pentanoate Modified $\mathrm{SiO}_{2}$ (SILCAs 9-12)

SILCAs 9-12 (see Table 1) were prepared based on the synthesis procedure for SILCA 2 with differentiation in terms of the base utilized. Instead of TMG the used bases were: 1,8-Diazabicyclo [5.4.0] undec-7-ene (SILCA 9), 1,5-Diazabicyclo[4.3.0]non-5-ene (SILCA 10), 1,5,7-Triazabicyclo [4.4.0]dec-5-ene (SILCA 11), and 2-Tert-Butyl-1,1,3,3-tetramethylguanidine (SILCA 12).

3.3.7. Synthesis of $\mathrm{Pd}(\mathrm{AcAc})_{2}, \mathrm{Pd}(\mathrm{OAc})_{2}$, and $\mathrm{Pd}\left(\mathrm{NO}_{3}\right)_{2}$ Supported on 3-Propyl Imidazolium Bromide-Tetramethylguanidinium Pentanoate Modified $\mathrm{SiO}_{2}$ (SILCAs 13-15)

SILCAs 13-15 (see Table 1) were prepared based on the synthesis procedure for SILCA 2 with variation of the palladium source. Instead of $\mathrm{PdCl}_{2}$ the used $\mathrm{Pd}$ was: $\mathrm{Pd}(\mathrm{AcAc})_{2}(\mathrm{SILCA} 13), \mathrm{Pd}(\mathrm{OAc})_{2}$ (SILCA 14), and $\mathrm{Pd}\left(\mathrm{NO}_{3}\right)_{2}$ (SILCA 15). 


\section{Conclusions}

In this work, a new group of catalysts was synthesized and investigated in order to develop a catalyst with a high activity and reusability in the Heck reaction. While performing the study, it became clear that correlating the catalyst activity, stability, and structure is a challenging task. Catalyst performance is hardly predictable and mostly depends on the ability to successfully graft different functionalities and obtain a durable layer which can stabilize Pd nanoparticles suppressing overgrowth and agglomeration. Material containing propyl imidazolium bromide-tetramethylguanidinium pentanoate modification gave a catalyst which preserved the activity in four successive cycles in the reaction of iodobenzene and butylacrylate at $100{ }^{\circ} \mathrm{C}$. It was demonstrated that the presence of carboxylic groups helps the coordination of tetramethylguanidine which is capable of stabilizing $\mathrm{Pd}$. The imidazole ring showed to be a rigid building unit vital for the ionic liquid layer formation, on the contrary to the aminoethylamino linker. However, this was not stable in the presence of bromosuccinate. It was found out that among the examined superbases, only TMG efficiently coordinates the acidic head of the auxiliary layer and that $\mathrm{PdCl}_{2}$ builds the most stable catalyst compared to other common Pd sources.

In summary, the catalyst with propyl imidazolium bromide-tetramethylguanidinium pentanoate resulted in a good metal distribution with controlled nanoparticle size and it showed a good thermal and chemical stability. This catalyst operates through the release and catch mechanism and, therefore, the use of it requires additional time for the metal re-deposition upon the end of the reaction. Slow leaching of palladium was observed which can be a consequence of catalyst poisoning by the by-products of Heck reaction and the solvents. Suppressing catalysts deactivation is an ongoing battle and further development of bis-layered catalysts have a potential to open a new field of studies to tackle this issue.

Supplementary Materials: The following are available online at http://www.mdpi.com/2073-4344/10/9/963/s1. Table S1: XPS data obtained for SILCAs 1-4; Table S2: XPS data obtained for SILCAs 5-8; Table S3: XPS data obtained for SILCAs 9-12; Table S4: XPS data obtained for SILCAs 13-15; Table S5: XPS data on Pd speciation for SILCAs 1-15; Figure S1: XPS Pd 3d spectral line of SILCA 1 after $5 \mathrm{~min}$ (a) and after $20 \mathrm{~min}$ (b) of measurements; Table S6: Representative TEM images of synthetized catalysts; Figure S2: Summarized isoelectric titration graphs for pristine $\mathrm{SiO}_{2}$ and SILCAs 1-12. Solution state ${ }^{1} \mathrm{H}$ and ${ }^{13} \mathrm{C}$ NMR of the esterified acids.

Author Contributions: Conceptualization, N.V., P.V., A.N., J.-P.M., and T.S.; methodology, N.V., P.V., J.-P.M., and T.S.; validation, N.V.; formal analysis, N.V., P.V., A.S., J.-P.M., and T.S.; investigation, N.V. and A.S.; data curation, N.V.; writing—original draft preparation, N.V.; writing—review and editing, N.V., P.V., A.S., J.-P.M., and T.S.; visualization, N.V.; supervision, P.V., J.-P.M., and T.S.; funding acquisition, J.-P.M. and T.S. All authors have read and agreed to the published version of the manuscript.

Funding: This research was funded by the Graduate School in Chemical Engineering (GSCE) of Finland and the Academy of Finland grants 319002, 320115, 325186, and 316827. This work is part of activities of the Technical Chemistry, Department of Chemistry, Chemical-Biological Centre, Umeå University, Sweden as well as the Johan Gadolin Process Chemistry Centre at Åbo Akademi University in Finland.

Acknowledgments: The Swedish Bio4Energy programme, Kempe Foundations, and Wallenberg Wood Science Center under auspices of Alice and Knut Wallenberg Foundation are gratefully acknowledged.

Conflicts of Interest: The authors declare no conflict of interest.

\section{References}

1. Yin, L.; Liebscher, J. Carbon-carbon coupling reactions catalyzed by heterogeneous palladium catalysts. Chem. Rev. 2007, 107, 133-173. [CrossRef]

2. Heck, R.F.; Nolley, J.P., Jr. Palladium-catalyzed vinylic hydrogen substitution reactions with aryl, benzyl, and styryl halides. J. Org. Chem. 1972, 37, 2320-2322. [CrossRef]

3. Mizoroki, T.; Mori, K.; Ozaki, A. Arylation of Olefin with Aryl Iodide Catalyzed by Palladium. Bull. Chem. Soc. Jpn. 1971, 44, 581. [CrossRef]

4. De Vries, J.G. The Heck reaction in the production of fine chemicals. Can. J. Chem. 2001, 79, 1086-1092. [CrossRef] 
5. Hübner, S.; De Vries, J.G.; Farina, V. Why Does Industry Not Use Immobilized Transition Metal Complexes as Catalysts? Adv. Synth. Catal. 2016, 358,3-25. [CrossRef]

6. Pagliaro, M.; Pandarus, V.; Ciriminna, R.; Béland, F.; Demma Carà, P. Heterogeneous versus Homogeneous Palladium Catalysts for Cross-Coupling Reactions. Chem CatChem 2012, 4, 432-445. [CrossRef]

7. Hagiwara, H.; Shimizu, Y.; Hoshi, T.; Suzuki, T.; Ando, M.; Ohkubo, K.; Yokoyama, C. Heterogeneous Heck reaction catalyzed by Pd/C in ionic liquid. Tetrahedron Lett. 2001, 42, 4349-4351. [CrossRef]

8. Corma, A.; García, H.; Leyva, A.; Primo, A. Basic zeolites containing palladium as bifunctional heterogeneous catalysts for the Heck reaction. Appl. Catal. A Gen. 2003, 247, 41-49. [CrossRef]

9. Dams, M.; Drijkoningen, L.; De Vos, D.E.; Jacobs, P.A.; Pauwels, B.; Van Tendeloo, G. Pd-zeolites as heterogeneous catalysts in Heck chemistry. J. Catal. 2002, 209, 225-236. [CrossRef]

10. Mpungose, P.P.; Vundla, Z.P.; Maguire, G.E.M.; Friedrich, H.B. The current status of heterogeneous palladium catalysed Heck and Suzuki cross-coupling reactions. Molecules 2018, 23, 1676. [CrossRef]

11. Grasa, G.A.; Singh, R.; Stevens, E.D.; Nolan, S.P. Catalytic activity of $\mathrm{Pd}(\mathrm{II})$ and $\mathrm{Pd}(\mathrm{II}) / \mathrm{DAB}-\mathrm{R}$ systems for the Heck arylation of olefins. J. Organomet. Chem. 2003, 687, 269-279. [CrossRef]

12. Köhler, K.; Heidenreich, R.G.; Krauter, J.G.E.; Pietsch, J. Highly active palladium/activated carbon catalysts for heck reactions: Correlation of activity, catalyst properties, and Pd leaching. Chem.-A Eur. J. 2002, 8, 622-631. [CrossRef]

13. Köhler, K.; Kleist, W.; Pröck, S.S. Genesis of coordinatively unsaturated palladium complexes dissolved from solid precursors during heck coupling reactions and their role as catalytically active species. Inorg. Chem. 2007, 46, 1876-1883. [CrossRef] [PubMed]

14. Reay, A.J.; Fairlamb, I.J.S. Catalytic C-H bond functionalisation chemistry: The case for quasi-heterogeneous catalysis. Chem. Commun. 2015, 51, 16289-16307. [CrossRef]

15. Gruttadauria, M.; Giacalone, F.; Noto, R. "Release and catch" catalytic systems. Green Chem. 2013, 15, 2608-2618. [CrossRef]

16. Aksin, Ö.; Türkmen, H.; Artok, L.; Çetinkaya, B.; Ni, C.; Büyükgüngör, O.; Özkal, E. Effect of immobilization on catalytic characteristics of saturated Pd-N-heterocyclic carbenes in Mizoroki-Heck reactions. J. Organomet. Chem. 2006, 691, 3027-3036. [CrossRef]

17. Gaikwad, A.V.; Rothenberg, G. In-situ UV-visible study of Pd nanocluster formation in solutionw. Phys. Chem. Chem. Phys. 2006. [CrossRef]

18. Reimann, S.; Stötzel, J.; Frahm, R.; Kleist, W.; Grunwaldt, J.-D.; Baiker, A. Identification of the Active Species Generated from Supported Pd Catalysts in Heck Reactions: An in situ Quick Scanning EXAFS Investigation. J. Am. Chem. Soc. 2011, 133, 3921-3930. [CrossRef]

19. Wanzlick, H.-W.; Schönherr, H.-J. Direct Synthesis of a Mercury Salt-Carbene Complex. Angew. Chemie Int. Ed. Engl. 1968, 7, 141-142. [CrossRef]

20. Öfele, K. 1,3-Dimethyl-4-imidazolinyliden-(2)-pentacarbonylchrom ein neuer übergangsmetall-carbenkomplex. J. Organomet. Chem. 1968, 12. [CrossRef]

21. Öfele, K.; Herrmann, W.A.; Mihalios, D.; Elison, M.; Herdtweck, E.; Scherer, W.; Mink, J. Mehrfachbindungen zwischen hauptgruppenelementen und übergangsmetallen. CXXVI. Heterocyclen-carbene als phosphananaloge liganden in metallkomplexen. J. Organomet. Chem. 1993, 459, 177-184. [CrossRef]

22. Herrmann, W.A.; Öfele, K.; Elison, M.; Kühn, F.E.; Roesky, P.W. Nucleophilic cyclocarbenes as ligands in metal halides and metal oxides. J. Organomet. Chem. 1994, 480. [CrossRef]

23. Weskamp, T.; Kohl, F.J.; Hieringer, W.; Gleich, D.; Herrmann, W.A. Highly Active Ruthenium Catalysts for Olefin Metathesis: The Synergy of N-Heterocyclic. Angew. Chemie Int. Ed. 1999, 39, 2416-2419. [CrossRef]

24. Schwarz, J.; Böhm, V.P.W.; Gardiner, M.G.; Grosche, M.; Herrmann, W.A.; Hieringer, W.; Raudaschl-Sieber, G. Polymer-Supported Carbene Complexes of Palladium: Well-Defined, Air-Stable, Recyclable Catalysts for the Heck Reaction. Chem.-A Eur. J. 2000, 6, 1773-1780. [CrossRef]

25. Gruttadauria, M.; Liotta, L.F.; Salvo, A.M.P.; Giacalone, F.; La Parola, V.; Aprile, C.; Noto, R. Multi-layered, covalently supported ionic liquid phase (mlc-SILP) as highly cross-linked support for recyclable palladium catalysts for the suzuki reaction in aqueous medium. Adv. Synth. Catal. 2011, 353, 2119-2130. [CrossRef]

26. Yang, H.; Han, X.; Li, G.; Wang, Y. N-Heterocyclic carbene palladium complex supported on ionic liquid-modified SBA-16: An efficient and highly recyclable catalyst for the Suzuki and Heck reactions. Green Chem. 2009, 11, 1184-1193. [CrossRef] 
27. Jung, J.Y.; Taher, A.; Kim, H.J.; Ahn, W.S.; Jin, M.J. Heck reaction catalyzed by mesoporous SBA-15-supported ionic liquid-Pd(OAc) $)_{2}$. Synlett 2009. [CrossRef]

28. Vucetic, N.; Virtanen, P.; Nuri, A.; Mattsson, I.; Aho, A.; Mikkola, J. Preparation and characterization of a new bis-layered supported ionic liquid catalyst (SILCA) with an unprecedented activity in the Heck reaction. J. Catal. 2019, 371, 35-46. [CrossRef]

29. Ji, Y.; Jain, S.; Davis, R.J. Investigation of Pd Leaching from Supported Pd Catalysts during the Heck Reaction. J. Phys. Chem. B 2005, 109, 17232-17238. [CrossRef]

30. Zhao, F.; Bhanage, B.M.; Shirai, M.; Arai, M. Heck Reactions of Iodobenzene and Methyl Acrylate with Conventional Supported Palladium Catalysts in the Presence of Organic and/or Inorganic Bases without Ligands. Chem.-A Eur. J. 2000, 6, 843-848. [CrossRef]

31. Thermo Scientific XPS: Knowledge Base. Available online: https://xpssimplified.com/periodictable.php (accessed on 27 February 2020).

32. X-ray Photoelectron Spectroscopy (XPS) Reference Pages. Available online: http://www.xpsfitting.com/ (accessed on 27 February 2020).

33. Moldoveanu, S.C.; David, V. Properties of Analytes and Matrices Determining HPLC Selection. In Selection of the HPLC Method in Chemical Analysis; Elsevier: Amsterdam, The Netherlands, 2017; pp. 189-230.

34. Amoroso, F.; Colussi, S.; Del Zotto, A.; Llorca, J.; Trovarelli, A. Room-temperature Suzuki-Miyaura reaction catalyzed by Pd supported on rare earth oxides: Influence of the point of zero charge on the catalytic activity. Catal. Lett. 2013, 143, 547-554. [CrossRef]

35. Hyde, A.M.; Calabria, R.; Arvary, R.; Wang, X.; Klapars, A. Investigating the Underappreciated Hydrolytic Instability of 1,8-Diazabicyclo [5.4.0] undec-7-ene and Related Unsaturated Nitrogenous Bases. Org. Process Res. Dev. 2019. [CrossRef]

36. Liu, X.; Mäki-Arvela, P.; Aho, A.; Vajglova, Z.; Gun'ko, V.M.; Heinmaa, I.; Kumar, N.; Eränen, K.; Salmi, T.; Murzin, D.Y. Zeta potential of beta zeolites: Influence of structure, acidity, $\mathrm{pH}$, temperature and concentration. Molecules 2018, 23, 946. [CrossRef] [PubMed]

37. National Institute of Advanced Industrial Science and Technology (AIST). Available online: https://www.aist. go.jp/index_en.html (accessed on 23 March 2020).

38. Potter, B.V.L.; Dowden, J.; Galione, A. Therapeutics Use of Pyridinium Compounds to Modulate Naadp Activity. WO 2005054198 A2, 16 June 2005.

(C) 2020 by the authors. Licensee MDPI, Basel, Switzerland. This article is an open access article distributed under the terms and conditions of the Creative Commons Attribution (CC BY) license (http://creativecommons.org/licenses/by/4.0/). 